\title{
The Problem of Conjunction and Disjunction in Quantum Logics
}

\author{
Jarosław Pykacz $^{1}$ (D) . Piotr Frąckiewicz ${ }^{2}$
}

Received: 29 December 2016 / Accepted: 22 April 2017 / Published online: 9 May 2017

(C) The Author(s) 2017. This article is an open access publication

\begin{abstract}
The long-lasting problem of proper mathematical representation of conjunctions and disjunctions in quantum logics is reviewed and three recent proposals of solutions are described.
\end{abstract}

Keywords Quantum logic · Conjunction · Disjunction · Orthomodular lattice

\section{Introduction}

The problem of finding proper mathematical representations of conjunction and disjunction in quantum logics is as old as the theory of quantum logics itself. In the LindenbaumTarski algebra of any theory governed by the laws of classical logic, which is a Boolean algebra, conjunctions and disjunctions of propositions are represented, respectively, by meets and joins. The same was assumed by Birkhoff and von Neumann [2] in the case of (ortho)modular lattices $\mathcal{L}(\mathcal{H})$ of closed linear subspaces of Hilbert spaces $\mathcal{H}$ that represent 'experimental propositions' pertaining to quantum objects.

However, Birkhoff and von Neumann were not entirely satisfied with this assumption, since they wrote:

It is worth remarking that in classical mechanics, one can easily define the meet or join of two experimental propositions as an experimental proposition - simply by having independent observers read off the measurements which either proposition involves, and

Jarosław Pykacz

pykacz@mat.ug.edu.pl

Piotr Frąckiewicz

P.Frackiewicz@impan.gov.pl

1 Institute of Mathematics, Department of Mathematics, Physics, and Informatics,

University of Gdańsk, Gdańsk, Poland

2 Institute of Mathematics, Pomeranian University, Słupsk, Poland 
combining the results logically. This is true in quantum mechanics only exceptionally - only when all the measurements involved commute (are compatible).

The significance of the problem of finding proper mathematical representations of conjunctions and disjunctions in $\mathcal{L}(\mathcal{H})$ follows from the fact that in numerous situations that are of utmost importance for contemporary research on foundations of quantum mechanics (EPR-type experiments, checking of Bell-type inequalities, etc.) there appear conjunctions of experimentally verifiable propositions that are represented by noncompatible elements of $\mathcal{L}(\mathcal{H})$.

Having in mind difficulties caused by unrestricted interpretation of meets and joins in $\mathcal{L}(\mathcal{H})$ as always representing conjunctions and disjunctions, in this paper we seek for other operations that would coincide with meets and joins on pairs of compatible elements of an orthomodular lattice, but could differ from them, or even be undefined, on pairs of elements that are not compatible.

\section{Basic Notions and Definitions}

In order to make the paper self-contained, we quote the necessary definitions. The reader more deeply interested in quantum logic theory is advised to acquaint with such milestone books as $[1,5,11]$. In this paper we use the name 'quantum logic' in a slightly narrower sense than usual, i.e., it will denote an orthomodular lattice, not an orthomodular poset, in this way staying closer to its very archetype: the orthomodular lattice of all (orthogonal projections onto) closed linear subspaces $\mathcal{L}(\mathcal{H})$ of an infinite-dimensional Hilbert space $\mathcal{H}$.

An orthomodular lattice (OML) is a lattice $L$ with $0_{L}$ and $1_{L}$ as the smallest and the greatest element, respectively, endowed with a unary operation $a \mapsto a^{\prime}$, called orthocomplementation, such that the following conditions are satisfied:

(i) $a^{\prime \prime}:=\left(a^{\prime}\right)^{\prime}=a$;

(ii) $a \leq b$ implies $b^{\prime} \leq a^{\prime}$;

(iii) $a \vee a^{\prime}=1_{L}$;

(iv) $a \leq b$ implies $b=a \vee\left(a^{\prime} \wedge b\right)$.

Condition (iv) is called the orthomodular law. If it is replaced by stronger distributivity laws:

$$
\begin{aligned}
& a \wedge(b \vee c)=(a \wedge b) \vee(a \wedge c) \\
& a \vee(b \wedge c)=(a \vee b) \wedge(a \vee c),
\end{aligned}
$$

then an OML becomes a Boolean algebra (BA). Elements of an OML are traditionally called propositions, since they are believed to represent experimentally verifiable propositions about properties of physical systems. If an OML $L$ is closed under countable lattice operations, then $L$ is called a $\sigma$-orthomodular lattice ( $\sigma$-OML). In the quantum logic approach to foundations of quantum mechanics, $\sigma$-OMLs are considered as mathematical models of sets of experimental propositions pertaining to quantum objects (see, e.g., $[1,11])$. In the traditional Hilbert space approach, this $\sigma$-OML is a lattice $\mathcal{L}(\mathcal{H})$ of all (orthogonal projections onto) closed linear subspaces of the corresponding Hilbert space $\mathcal{H}$.

Let $L$ be an OML. Two elements $a, b \in L$ are called orthogonal (denoted $a \perp b$ ) iff $a \leq b^{\prime}$, and $a, b$ are called compatible (denoted $a \leftrightarrow b$ ) iff there exist in $L$ three pairwisely orthogonal elements $a_{1}, b_{1}, c$ such that $a=a_{1} \vee c$ and $b=b_{1} \vee c$. It occurs that in such a case $a_{1}=a \wedge b^{\prime}, b_{1}=b \wedge a^{\prime}$, and $c=a \wedge b$, and it is said that the triple $\left\{a_{1}, b_{1}, c\right\}$ forms the Mackey decomposition of a pair of compatible propositions $\{a, b\}[7,14,19]$. Notice 
that two projections on a Hilbert space are orthogonal iff the product of them is zero, and compatible iff they commute. In Boolean algebras all elements are compatible.

A probability measure on $L$ is a mapping $s: L \rightarrow[0,1]$ such that

(i) $s\left(1_{L}\right)=1$;

(ii) $a \perp b$ implies $s(a \vee b)=s(a)+s(b)$.

Probability measures on OMLs are also called states since in the quantum logic approach to foundations of quantum mechanics they actually represent states of studied physical objects. According to the standard interpretation a number $s(a)$ is a probability that when a studied physical object is in a state represented by $s$, then an experiment designed to check whether an experimental proposition represented by $a$ is true, will yield positive result. However, we shall argue in Section 4 that also other interpretation of this number is possible.

A set of states $S$ on an OML $L$ is called ordering iff for all $s \in S$ and all $a, b \in L$, $s(a) \leq s(b)$ implies $a \leq b$. Let us note that from the physical point of view only OMLs with ordering sets of states are interesting, since the only way to establish experimentally partial order relation between experimental propositions is to make a series of experiments on the studied physical object prepared in various states, checking in this way whether $s(a) \leq s(b)$ for various states $s$.

\section{Conjunctions and Disjunctions Generated by Kotas Conditionals}

In a paper [14] one of the authors proposed to use a formal Mackey decomposition: $\left\{a_{1}, b_{1}, c\right\}$, where $a_{1}=a \wedge b^{\prime}, b_{1}=b \wedge a^{\prime}$, and $c=a \wedge b$, of any two (not necessarily compatible) elements $a, b \in \mathrm{OML}$ in order to define the following conjunction-like and disjunction-like operations on an OML:

$$
\begin{aligned}
& a \sharp b:=a_{1} \vee b_{1} \vee c=\left(a \wedge b^{\prime}\right) \vee\left(b \wedge a^{\prime}\right) \vee(a \wedge b) \\
& a b b:=\left(a^{\prime} \sharp b^{\prime}\right)^{\prime}=\left(a \vee b^{\prime}\right) \wedge\left(b \vee a^{\prime}\right) \wedge(a \vee b) .
\end{aligned}
$$

It occurs that for all $a, b \in \mathrm{OML}$ :

$$
a \leftrightarrow b \quad \text { iff } \quad a \sharp b=a \vee b \quad \text { and } \quad a b b=a \wedge b,
$$

so the operations $\sharp$ and $b$ fulfill the requirement stated in the Introduction. This also means that they satisfy numerous identities that are typical to conjunction and disjunction (idempotency, law of excluded middle, law if contradiction, $0-1$ laws, etc.). Moreover, they define on an OML partial order relation in the same way as meets and joins do:

$$
a \preceq b \quad \text { iff } \quad a b b=a \quad \text { iff } \quad a \sharp b=b,
$$

and this relation coincides with the original partial order:

$$
a \preceq b \quad \text { iff } \quad a \leq b \quad \text { for any } a, b \in \text { OML. }
$$

Later on the late Secretary of the International Quantum Structures Association Dr. Bart D'Hooghe found that the pair of operations $\sharp$ and $b$ is one of five possible pairs of operations, different from join and meet, that can be defined by the so called Kotas conditionals [6] and orthocomplementation in the same way as disjunction and conjunction can be defined by implication and negation in classical logic: $a$ OR $b:=$ (NOT $a$ ) IMPLIES $b$, $a \mathrm{AND} b:=$ NOT ((NOT $a)$ OR (NOT $b))$. These operations, expressed with the aid of meet, 
join, and orthocomplementation, are as follows (the numbering is the same as original Kotas numbering of his conditionals in [6]):

$$
\begin{aligned}
& a \sharp_{1} b:=a \vee\left(a^{\prime} \wedge b\right) \\
& a \sharp_{2} b:=\left(a \wedge b^{\prime}\right) \vee b \\
& a \sharp_{3} b:=\left(a \wedge b^{\prime}\right) \vee\left(b \wedge a^{\prime}\right) \vee(a \wedge b) \\
& a \sharp_{4} b:=\left(a^{\prime} \wedge b\right) \vee(a \wedge b) \vee\left((a \vee b) \wedge b^{\prime}\right) \\
& a \sharp_{5} b:=\left(a^{\prime} \wedge(a \vee b)\right) \vee(a \wedge b) \vee\left(a \wedge b^{\prime}\right),
\end{aligned}
$$

and $a b_{i} b:=\left(a^{\prime} \sharp_{i} b^{\prime}\right)^{\prime}$ for $i=1, \ldots, 5$.

Obviously operations $\sharp$ and $b$ defined previously without reference to the third Kotas conditional coincide, respectively, with $\sharp_{3}$ and $b_{3}$. It is also worth noticing that only operations $\sharp_{3}$ and $b_{3}$ are, like join and meet, commutative on both: compatible and noncompatible elements of an OML. Operations $\sharp_{i}$ and $b_{i}$ for $i=1,2,4,5$ are not commutative on noncompatible elements of an OML. Except of commutativity, all operations generated by Kotas conditionals have the same properties [3]. ${ }^{1}$ In particular they all have property expressed in (3), i.e., they all coincide with join and meet on and only on compatible elements of an OML, and they all define by formula (4) partial order relation that coincides with the original one. This means that all these pairs of operations fulfill the requirement stated in Introduction, and that it is not possible to state experimentally which one of them is the proper mathematical representation of conjunctions and disjunctions in quantum logics since one can simultaneously check experimental propositions if and only if they are represented by compatible elements of an OML.

\section{Conjunctions and Disjunctions in Lukasiewicz $\infty$-valued Representation of Quantum Logics}

The results of investigations performed by one of the authors through years [12, 13, 15, 16] (all results are collected in [17]) show that every OML (even orthomodular poset) with an ordering set of states $S$ can be isomorphically represented as a family $\mathbb{L}(S)$ of infinite-valued propositional functions defined on $S$ endowed with Lukasiewicz conjunction:

$$
[a(\cdot) \sqcap b(\cdot)]:=\max ([a(\cdot)]+[b(\cdot)]-1,0),
$$

where $[a(s)] \in[0,1]$ denotes truth-value of a proposition $a(s)$ obtained from the propositional function $a(\cdot)$ by inserting into it a (name of) a state $s$, and Lukasiewicz disjunction:

$$
[a(\cdot) \sqcup b(\cdot)]:=\min ([a(\cdot)]+[b(\cdot)], 1),
$$

such that:

(a) The always-false propositional function $f(\cdot)$ (i.e., such function that $\forall s \in S$, $[f(s)]=0)$ belongs to $\mathbb{L}(S)$.

(b) $\mathbb{L}(S)$ is closed with respect to Eukasiewicz negation $[\neg a(\cdot)]:=1-[a(\cdot)]$.

(c) If $\left\{a_{i}(\cdot)\right\}$ is a sequence of propositional functions such that $a(\cdot)_{i} \sqcap a(\cdot)_{j}=f(\cdot)$

for $i \neq j$ (such propositional functions are called exclusive), then $\sqcup_{i} a_{i}(\cdot) \in \mathbb{L}(S)$.

\footnotetext{
${ }^{1}$ Properties of operations $\sharp_{i}$ and $b_{i}$ were listed in [3] without proofs. Proofs were supposed to be published in [4], which never happened due to untimely death of Dr. Bart D'Hooghe.
} 
(d) The always-false propositional function $f$ is the only propositional function in $\mathbb{L}(S)$ that is exclusive with itself, i.e., for any $a(\cdot) \in \mathbb{L}(S)$, if $a(\cdot) \sqcap a(\cdot)=f(\cdot)$, then $a(\cdot)=f(\cdot)$.

Partial order relation in $\mathbb{L}(S)$ is defined by Łukasiewicz implication:

$$
[a(\cdot) \rightarrow b(\cdot)]:=\min (1-[a(\cdot)]+[b(\cdot)], 1)
$$

via the formula:

$$
a(\cdot) \leq b(\cdot) \quad \text { iff } \quad a(\cdot) \rightarrow b(\cdot)=t(\cdot),
$$

where $t(\cdot)$ denotes the always-true propositional function, and we infer from (13) that $a(\cdot) \leq$ $b(\cdot)$ iff $a(s) \leq b(s) \forall s \in S$.

The isomorphism between an OML $L$ with an ordering set of states $S$ and the family of propositional functions $\mathbb{L}(S)$ satisfying conditions (a) - (d) is defined as follows:

$$
\forall a \in L \quad a \mapsto a(\cdot) \quad \text { s.t. } \forall s \in S \quad a(s)=s(a) .
$$

It occurs that for any state $s \in S$ and any experimental proposition represented by an element $a$ of an OML a number $s(a)=a(s)$ usually interpreted as the probability of getting positive result in an experiment designed to check whether $a$ is true when a studied physical object is in a state represented by $s$, can be reinterpreted as a truth-value of an infinite-valued proposition "an experiment designed to check " $a$ " when a physical system is in the state " $s$ " will yield positive result'. It should be stressed that this proposition concerns future event the result of not-yet-performed experiment. When the experiment is completed we are back to classical two-valued logic, which is a source of an erroneous conviction that quantum logic, although non-classical because of non-distributivity, is two-valued (cf. [15]).

\section{1 Łukasiewicz Conjunction and Disjunction Versus Meet and Join}

Because of the isomorphism mentioned in the beginning of this Section, we have two pairs of conjunction-like and disjunction-like operations on an OML with an ordering set of states $S$ isomorphically represented by the family $\mathbb{L}(S)$ : meets and joins generated by partial order relation (14), and Łukasiewicz conjunctions and disjunctions. Of course it is very interesting to study relations between these two pairs of operations, specially to check whether Łukasiewicz operations could be used as models of conjunctions and disjunctions in OMLs instead of meets and joins.

The following theorem was proved in a different and slightly more general form (fuzzy sets instead of propositional functions, and OMPs instead of OMLs) in [13]:

Theorem 1 Let $L$ be an OML with an ordering set of states $S$ and let $\mathbb{L}(S)$ be its isomorphic representation as a family of $\infty$-valued propositional functions defined on $S$. Let a $(\cdot), b(\cdot) \in$ $\mathbb{L}(S)$. Then $a(\cdot) \sqcap b(\cdot) \in \mathbb{L}(S)$ iff $a(\cdot) \sqcup b(\cdot) \in \mathbb{L}(S)$, and in this case $a(\cdot)$ and $b(\cdot)$ are compatible, $a(\cdot) \sqcap b(\cdot)=a(\cdot) \wedge b(\cdot)$, and $a(\cdot) \sqcup b(\cdot)=a(\cdot) \vee b(\cdot)$.

From this theorem it is clearly visible that Łukasiewicz conjunctions and disjunctions are, in general, only partially defined. Indeed, if they were globally defined, then any two elements would be compatible, so any OML with an ordering set of states would be a Boolean algebra.

By transposition of an implication $a(\cdot) \sqcap b(\cdot), a(\cdot) \sqcup b(\cdot) \in \mathbb{L}(S) \Rightarrow a(\cdot) \leftrightarrow b(\cdot)$ we obtain Corollary:

Corollary 1 If $a(\cdot), b(\cdot) \in \mathbb{L}(S)$ are not compatible, then $a(\cdot) \sqcap b(\cdot), a(\cdot) \sqcup b(\cdot)$ are not defined in $\mathbb{L}(S)$. 
Theorem 1 and Corollary 1 mean that Łukasiewicz conjunction and disjunction 'almost' fulfill requirements stated in Introduction: although they are only partially defined on $\mathbb{L}(S)$, when they are defined they coincide, respectively, with meet and join, and in this case all pairs of elements on which they are defined are compatible. On the other hand, they are not defined on noncompatible elements of $\mathbb{L}(S)$ which, since experimental propositions can be checked simultaneously only when they are represented by compatible elements of an OML, causes no problems. Therefore, it is very tempting to state that conjunctions and disjunctions in quantum logic should not be modeled by meets and joins but by Łukasiewicz conjunctions and disjunctions, which coincide with meets and joins whenever they are defined.

Unfortunately, in $[13,17]$ there were given examples of Boolean algebras in which Łukasiewicz conjunctions and disjunctions were not defined on some pairs of elements. This shows that compatibility of elements is not a sufficient condition for Łukasiewicz operations to be defined on them. However, it should be stressed that examples studied in [13, 17] are highly artificial and it is not known yet whether such examples could be found in the case of an OML $\mathcal{L}(\mathcal{H})$ of (orthogonal projections onto) closed linear subspaces of a Hilbert space $\mathcal{H}$ that represent 'experimental propositions' pertaining to a quantum object.

The other unpleasant feature of Łukasiewicz conjunction and disjunction is their nonidempotency: If for some state $s \in S$ the truth value of a proposition $a(s)$ is non-classical, i.e., $[a(s)] \notin\{0,1\}$, then $[a(s) \sqcap a(s)]=\max (2[a(s)]-1,0) \neq[a(s)]$ and $[a(s) \sqcup a(s)]=$ $\min (2[a(s)], 1) \neq[a(s)]$. However, this drawback could be neglected since conjunction or disjunction of a proposition with itself is very strange even in daily speech, so such cases could be eliminated 'by default'.

\section{$5 s$-Maps and $j$-Maps as Probabilities of Conjunctions and Disjunctions}

The third proposal of solving the problem of conjunctions and disjunctions in quantum logic is different from two previous ones. The aim of two previous proposals was to find new operations on OMLs that coincide with meets and joins on pairs of compatible elements but would be either different from meets and joins, or be not defined at all on pairs of elements that are not compatible. However, in mathematical description of quantum systems we are usually not so much interested what element of an OML $\mathcal{L}(\mathcal{H})$ represents conjunction or disjunction of two experimentally verifiable propositions, but rather what is the probability that their conjunction or disjunction is true. Therefore, it would be sufficient to define on the Cartesian product of an OML with itself functions of two variables that would coincide with a probability of meet or join on pairs of compatible elements but could be different from them when their arguments are noncompatible. This was exactly the aim of O. Nánásiová when she introduced in [8] the notion of a map for simultaneous measurements (abbr. s-map) on an OML as a function $p: L \times L \rightarrow[0,1]$ such that:

(s1) $p\left(1_{L}, 1_{L}\right)=1$;

(s2) if $a \perp b$, then $p(a, b)=0$;

(s3) if $a \perp b$, then for any $c \in L$ :

$$
\begin{aligned}
& p(a \vee b, c)=p(a, c)+p(b, c), \\
& p(c, a \vee b)=p(c, a)+p(c, b) .
\end{aligned}
$$

It occurs that each s-map $p$ generates on an OML a probability measure $s_{p}$ by the formula $s_{p}(a)=p(a, a)=p\left(1_{L}, a\right)=p\left(a, 1_{L}\right)$ and that for any two compatible propositions $p(a, b)=s_{p}(a \wedge b)$. This means that in the case of compatible propositions the number 
$p(a, b)$ can be really thought of as representing probability of simultaneous verification of both propositions in a single experiment. If propositions $a$ and $b$ are not compatible, $p(a, b)$ may be different from $s_{p}(a \wedge b)$. Nevertheless, the value $p(a, b)$ can be thought of as representing probability of simultaneous verification of $a$ and $b$ in a 'counterfactual measurement', so one could say: ' $p(a, b)$ is the probability of simultaneous verification of propositions $a$ and $b$ if we were able perform it' or, equivalently, the truth-value of 'counterfactual conjunction' of propositions $a$ and $b$.

In [10] (see also [9], [18]) the following notion of a join map (abbr. $j$-map) on an OML as a function $q: L \times L \mapsto[0,1]$ was introduced:

(j1) $q\left(0_{L}, 0_{L}\right)=0, \quad q\left(1_{L}, 1_{L}\right)=1$;

(j2) if $a \perp b$, then $q(a, b)=q(a, a)+q(b, b)$;

(j3) if $a \perp b$, then for any $c \in L$ :

$$
\begin{aligned}
& q(a \vee b, c)=q(a, c)+q(b, c)-q(c, c) \\
& q(c, a \vee b)=q(c, a)+q(c, b)-q(c, c) .
\end{aligned}
$$

It was proved in [9] that if $p$ is an $s$-map on an OML, then $q_{p}(a, b)=p(a, a)+$ $p(b, b)-p(a, b)=s_{p}(a)+s_{p}(b)-p(a, b)$ is a $j$-map. It is easy to see that if $a \leftrightarrow b$, then $q_{p}(a, b)=s_{p}(a)+s_{p}(b)-s_{p}(a \wedge b)=s_{p}(a \vee b)$, so again for compatible propositions this number can be thought of as probability of verification of disjunction ' $a$ or $b$ ' in a single experiment. If propositions $a$ and $b$ are not compatible, this number can be thought of as representing probability of verification of disjunction ' $a$ or $b$ ' in a 'counterfactual measurement' or, equivalently, the truth-value of 'counterfactual disjunction' of propositions $a$ and $b$.

\section{Summary and Open Questions}

We proposed three solutions to the long-lasting problem of finding proper mathematical representations of conjunctions and disjunctions in quantum logics. Although according to Birkhoff and von Neumann [2] in the case of compatible propositions their conjuctions and disjunctions are properly modeled by their meets and joins, extending this statement to propositions that are not compatible is very doubtful.

Since simultaneous verification of experimental propositions that are represented by noncompatible elements of an OML $\mathcal{L}(\mathcal{H})$ is not possible, the problem which of the proposed operations are better models of conjunctions and disjunctions in $\mathcal{L}(\mathcal{H})$ can be settled only with the aid of theoretical arguments. In this respect finding answers to open questions listed below could be of some help:

Q1. Are there any links between proposals presented in Sections 3, 4, and 5?

Q2. Can Łukasiewicz conjunction and disjunction be not defined on two compatible elements of $\mathcal{L}(\mathcal{H})$ ? If not - what specific properties of $\mathcal{L}(\mathcal{H})$ cause this?

Q3. Let $s$ be a probability measure on an OML. Is it always possible to construct an s-map $p$ such that $s=s_{p}$ ?

Acknowledgments Work by Piotr Frąckiewicz was supported by the Ministry of Science and Higher Education in Poland under the project Iuventus Plus IP2014 010973 in the years 2015-2017. 
Open Access This article is distributed under the terms of the Creative Commons Attribution 4.0 International License (http://creativecommons.org/licenses/by/4.0/), which permits unrestricted use, distribution, and reproduction in any medium, provided you give appropriate credit to the original author(s) and the source, provide a link to the Creative Commons license, and indicate if changes were made.

\section{References}

1. Beltrametti, E., Cassinelli, G.: The Logic of Quantum Mechanics. Addison-Wesley, Reading, MA (1981)

2. Birkhoff, G., Von Neumann, J.: The logic of quantum mechanics. Ann. Math. 37, 823-843 (1936)

3. D'Hooghe, B., Pykacz, J.: On some new operations on orthomodular lattices. Int. J. Theor. Phys. 39, 641-652 (2000)

4. D'Hooghe, B.: New operations on orthomodular lattices II. 'Disjuntions' and 'conjunctions' generated by Kotas conditionals. Unpublished manuscript (2000)

5. Engesser, K., Gabbay, D., Lehmann, D. (eds.): Handbook of Quantum Logic and Quantum Structures. Elsevier, Amsterdam (2007)

6. Kotas, J.: An axiom system for the modular logic. Stud. Logica 21, 17-38 (1967)

7. Mackey, G.W.: The Mathematical Foundations of Quantum Mechanics. Benjamin, New York (1963)

8. Nánásiová, O.: Map for simultaneous measurements for a quantum logic. Int. J. Theor. Phys. 42, 18891903 (2003)

9. Nánásiová, O., Valášková, L.: Maps on a quantum logic. Soft Comput. 14, 1047-1052 (2010)

10. Nánásiová, O., Minárová, M., Mohammed, A.: Probability and quantum logic. Forum Statisticum Slovacum 5/2007, 101-107 (2007)

11. Pták, P., Pulmannová, S.: Orthomodular Structures as Quantum Logics. Kluwer, Dordrecht (1991)

12. Pykacz, J.: Fuzzy quantum logic and infinite-valued Łukasiewicz logic. Int. J. Theor. Phys. 33, 14031416 (1994)

13. Pykacz, J.: Łukasiewicz operations in fuzzy set and many-valued representations of quantum logics. Found. Phys. 30, $1503-1524$ (2000)

14. Pykacz, J.: New operations on orthomodular lattices: 'conjunction' and 'disjunction' induced by Mackey decompositions. Notre Dame J. Form. Logic 41, 59-76 (2000)

15. Pykacz, J.: Unification of two approaches to quantum logic: Every Birkhoff - von Neumann quantum logic is a partial infinite-valued Łukasiewicz logic. Stud. Logica 95, 5-20 (2010)

16. Pykacz, J.: Towards many-valued/fuzzy interpretation of quantum mechanics. Int. J. Gen. Syst. 40, 11-21 (2011)

17. Pykacz, J.: Quantum Physics, Fuzzy Sets and Logic. Steps Towards a Many-Valued Interpretation of Quantum Mechanics. Springer, Cham (2015)

18. Pykacz, J., Valášková, L., Nánásiová, O.: Bell-type inequalities for bivariate maps on orthomodular lattices. Found. Phys. 45, 900-913 (2015)

19. Younce, M.B.: Refinement and unique Mackey decomposition for manuals and orthoalgebras. Found. Phys. 20, 691-700 (1990) 